\title{
Speed enhancement of magnetic logic-memory device by insulator-to-metal transition 중
}

\author{
Cite as: Appl. Phys. Lett. 117, 022407 (2020); doi: 10.1063/5.0013301 \\ Submitted: 11 May 2020 - Accepted: 19 June 2020 . \\ Published Online: 14 July 2020
}

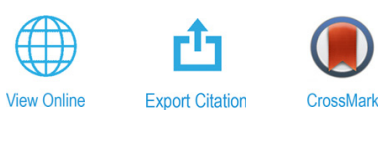

Yuchen Pu, ${ }^{7}$ Hongming Mou, Ziyao Lu, ${ }^{7}$ Seeraz Nawaz, ${ }^{1}$ Guilin Wang, ${ }^{2}$ Zhigang Zhang, ${ }^{3}$ Yuanjun Yang, ${ }^{2}$ Xixiang Zhang, ${ }^{4}$ (D) and Xiaozhong Zhang ${ }^{1, a)}$ (iD)

\author{
AFFILIATIONS \\ ${ }^{1}$ Key Laboratory of Advanced Materials (MOE), School of Materials Science and Engineering, Tsinghua University, Beijing 100084, \\ China \\ ${ }^{2}$ Department of Physics, and Lab of Solid State Microstructures and Quantum Control, School of Electronic Science and Applied \\ Physics, Hefei University of Technology, Hefei, Anhui 230009, People's Republic of China \\ ${ }^{3}$ Institute of Microelectronics, Tsinghua University, Beijing 100084, China \\ ${ }^{4}$ Division of Physical Science and Engineering, King Abdullah University of Science and Technology (KAUST), Thuwal 239955, \\ Kingdom of Saudi Arabia
}

${ }^{a)}$ Author to whom correspondence should be addressed: xzzhang@tsinghua.edu.cn

\begin{abstract}
Complementary metal-oxide-semiconductor logic circuits used in conventional computers require frequent communication with external nonvolatile memory, causing the memory wall problem. Recently reported magnetic logic with reconfigurable logic operation and built-in nonvolatile memory can potentially bridge this gap. However, its high-frequency performance is not well studied. Here, we first perform experimental and theoretical investigation on the switching time of magnetic logic-memory devices combining magnetic units and negative differential resistance (NDR) of semiconductors. It is found that the switching time of S-type NDR (transistor circuits) in logic operations is $\sim 300 \mathrm{~ns}$ and determined by the transistor's internal turn-on properties. We then propose a magnetic logic-memory device by coupling the anomalous Hall effect in magnetic materials and the insulator-to-metal transition in $\mathrm{VO}_{2}$. Our device realizes reliable output (output ratio $>1000 \%)$, a low work magnetic field $(<20 \mathrm{mT})$, and excellent high-frequency performance (switching time $=1-10 \mathrm{~ns}$ ).
\end{abstract}

Published under license by AIP Publishing. https://doi.org/10.1063/5.0013301

Conventional computers consist of volatile silicon-based complementary metal-oxide-semiconductor (CMOS) logic and external nonvolatile memory. With this Von Neumann architecture, frequent communication between the logic processor and the memory is required, causing the memory wall problem. ${ }^{1}$ Luo et al. ${ }^{2}$ proposed a magnetic logic-memory device by the use of the anomalous Hall effect (AHE) in magnetic materials and the negative differential resistance (NDR) in semiconductors, realizing a magnetic logic with both a high logic output ratio $\left(>10^{4} \%\right)$ and a low work magnetic field $(<50 \mathrm{mT})$. This logic-memory device overcomes the disadvantage of the spinbased $\operatorname{logic}^{3}$ and the magnetic field-based logic. ${ }^{4}$ Besides, non-volatile information reading, processing, and writing could be reliably realized in one step and in one simple device. The time and energy used in the processes of information transformation and transfer could be saved so that the performance of energy consumption and operation speed could be improved.

In Luo's magnetic logic-memory device, a current-controlled Stype $\mathrm{NDR}^{5}$ consists of bipolar junction transistor (BJT) pairs and a linear resistor. The S-typed NDR is conventionally used in analog circuits. It is not known if the S-type NDR has good high-frequency performance in logic operations, i.e., the switching time of the magnetic logic-memory device is not well studied. In this work, we present experimental and theoretical studies on the switching time of the device and find that the switching time of the S-type NDR in logic operations is significantly longer than that of CMOS logic. To improve the highfrequency performance of the logic-memory device, it is necessary to solve the problem of long switching time of the S-type NDR in logic operations. In insulator-to-metal transition (IMT) materials, ${ }^{6}$ e.g., $\mathrm{VO}_{2}$, an abrupt decrease in resistivity is triggered when the current reaches a critical current threshold. ${ }^{8}$ This makes it possible that the IMT materials can act as a NDR with a short switching time in logic operations. ${ }^{9}$ By coupling the AHE in magnetic materials and the IMT in $\mathrm{VO}_{2}$, we propose a magnetic logic-memory device with reliable output, a low work magnetic field, and excellent high-frequency performance.

The thermally oxidized $\mathrm{Si}$ wafers with a $\mathrm{SiO}_{2}$ layer $(300 \mathrm{~nm})$ are used as substrates for magnetic thin films. Magnetic multilayer films 
with the structure of $\mathrm{Ta}(3.5 \mathrm{~nm}) / \mathrm{CoFeB}(1 \mathrm{~nm}) / \mathrm{MgO}(1.3 \mathrm{~nm})$ are prepared using a sputter system (Rotaris, Singulus). The samples are deposited, at room temperature, with the base pressure lower than $8.0 \times 10^{-9}$ mbar. The deposition of $\mathrm{SiO}_{2}$ with a thickness of $5 \mathrm{~nm}$ as a top layer for each sample is carried out to prevent sample oxidization. During deposition, the gas atmosphere is Argon with a flow rate of 55 $\mathrm{sccm}$ and the process pressure was $3 \times 10^{-3} \mathrm{mbar}$. All samples are annealed at $300^{\circ} \mathrm{C}$ for one hour in vacuum $\left(<5 \times 10^{-7} \mathrm{mbar}\right)$ to achieve good perpendicular magnetic anisotropy (PMA). To fabricate the three-terminal magnetic component, magnetic multilayer films are patterned using photolithography and then Ar-ion milling. Before depositing $\mathrm{Ti}(10 \mathrm{~nm}) / \mathrm{Au}(50 \mathrm{~nm})$ electrodes, slight plasma cleaning is employed to remove the $\mathrm{SiO}_{2}$ protective layer and make a good electrical contact.

Due to the AHE of magnetic material, electrons with different spin directions are deflected in opposite directions, which causes an imbalance of the spin-polarized electron current flowing left and right [Fig. 1(a)]. This asymmetry could be used to perform a reconfigurable logic, but the output ratio based on the pure anomalous Hall effect is only $2 \%$ [Fig. 1(b)]. In order to improve output performance, Luo et $a .^{2}$ introduced NDR components into the magnetic device [Figs. 1(c) and 1(f)]. As shown in Fig. 1(d), an extremely large magnetismcontrolled current bifurcation is observed. Left and right channels are, therefore, in a high current state (HCS) and a low current state (LCS), respectively, and $\mathrm{I}_{\text {Left }} / \mathrm{I}_{\text {Right }}$ could be $>10^{4} \%$. The magnetic memory bit connecting the HCS channel could be switched by the spin-orbit torques (SOT), so that the information was written in it, which makes this device a nonvolatile logic-memory device.

We first experimentally investigate the switching time of the NDR component in logic operations. The measurement is performed using an oscilloscope at room temperature $(300 \mathrm{~K})$ as shown in Fig. 1(e). A current/voltage pulse is applied from a pulse generator to the device and a resistor $\mathrm{R}_{\mathrm{m}}$. Voltages of Channel $1(\mathrm{CH} 1)$ and Channel 2 $(\mathrm{CH} 2)$ indicate the input and output signals through the device, respectively, and the switching time is, therefore, the delay time between these two channels.

As shown in Figs. 2(a)-2(c), the range of NDR switching times is $42-468 \mathrm{~ns}$ with the variation of $\mathrm{R}_{\mathrm{NDR}}$ and applied current pulses. For a NDR with amplifier BJT pairs and switching BJT pairs, the switching time increases rapidly and then remains stable with the increase in $\mathrm{R}_{\mathrm{NDR}}$ when the applied current pulse is fixed. However, for a NDR with wideband BJT pairs, the switching time is insensitive to $R_{N D R}$. In order to investigate the extent of $\mathrm{R}_{\mathrm{NDR}}$ and BJT pairs' influences on the switching time, we compare the NDR switching time with different BJT pairs [Fig. 2(d)]. If a current pulse of $2 \mathrm{~mA}$ is applied, the difference in the switching time among the NDR with different BJT pairs is $41.2 \%$ when $\mathrm{R}_{\mathrm{NDR}}$ is $100 \Omega$, which is 3 times larger than the difference when $\mathrm{R}_{\mathrm{NDR}}$ is $880 \Omega(12.1 \%)$. Hence, the internal property of complemental BJT pairs determines the switching time in small $\mathrm{R}_{\mathrm{NDR}}$, while it has a little influence on the switching time in larger $\mathrm{R}_{\mathrm{NDR}}$.

The measured switching time of S-type NDR is several hundred nanoseconds, much greater than that of SOT and CMOS circuits. Our experimental results indicate that the enlarged current bifurcation in our magnetic logic device cannot be observed if $\mathrm{R}_{\mathrm{NDR}}<90 \Omega$, which corresponds to Shoucair's theory. ${ }^{10}$ Hence, we cannot bring down the switching time by further reduction of $\mathrm{R}_{\mathrm{NDR}}$. Moreover, even if $\mathrm{R}_{\mathrm{NDR}}$ $=0$, the measured switching time is $37.8 \mathrm{~ns}$. Therefore, in order to
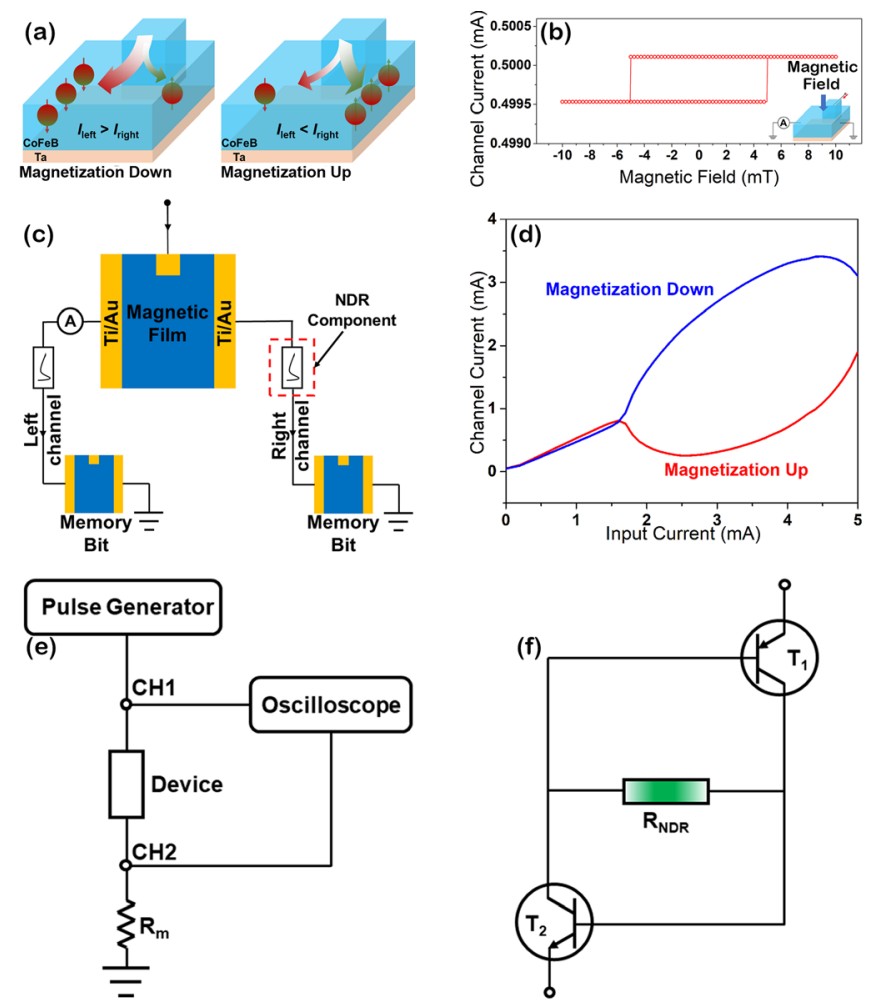

FIG. 1. (a) Schematics of the anomalous Hall effect of magnetization down (left) and up (right). (b) Anomalous Hall effect-induced current imbalance of a $\mathrm{MgO} /$ $\mathrm{CoFeB} / \mathrm{Ta}$ multilayer film. The applied current is $1 \mathrm{~mA}$; the inset shows the configuration of measurements. (c) Schematics of the magnetic logic-memory device structure. (d) Left channel current of magnetization down and up based on the structure shown in (c). (e) System for the switching time measurement. In the measurement of the NDR component, the current pulses with a $5 \mu \mathrm{s}$ width and $1.5 \mu$ s increasing time are applied, and the current varies from $2 \mathrm{~mA}$ to $5 \mathrm{~mA}$. In the measurement of the two-terminal $\mathrm{VO}_{2}$ device, the width and increasing time of applied voltage pulse are 10 and $1 \mathrm{~ns}$, and the current varies from $0.7 \mathrm{~mA}$ to $3 \mathrm{~mA}$. (f) Circuit of the Stype NDR made of complementary BJT pairs $\left(p-n-p\right.$ transistor $T_{1}$ and $n-p-n$ transistor $T_{2}$ ) and a linear resistor $\left(R_{N D R}\right)$. Three types of complementary BJT pairs are used: amplifier BJT pairs, wideband BJT pairs, and switching BJT pairs.

find the minimum switching time that could be achieved, we develop a physical model based on the nonlinear lumped theory ${ }^{11}$ (the details can be seen in the supplementary material). The results obtained from the model show that both BJT pairs' internal turn-on properties and the linear resistor have an influence on the switching time of the NDR, and so the minimum switching time in logic operations of the semiconductor NDR is $\sim 30$ ns. Therefore, it is necessary to replace the NDR by a more suitable device to achieve better high-frequency performance, such as the IMT device made of $\mathrm{VO}_{2}$ material used in this work.

The $\mathrm{VO}_{2}$ thin films are grown in an epitaxial manner on (0001)$\mathrm{Al}_{2} \mathrm{O}_{3}$ substrates using the RF-magnetron sputtering technique. A 2in. vanadium metal target is sputtered at an RF power of $65 \mathrm{~W}$. The pure argon and oxygen flow ratios are 50 and $1.5 \mathrm{sccm}$ (standard cubic centimeters per minute), respectively. The sputtering pressure is kept at $\sim 0.43 \mathrm{~Pa}$. The growth temperature is $540{ }^{\circ} \mathrm{C}$. The substrate holder is self-rotated to ensure good uniformity of the $\mathrm{VO}_{2}$ thin films. For the two-terminal device, the channel width and isolation are 

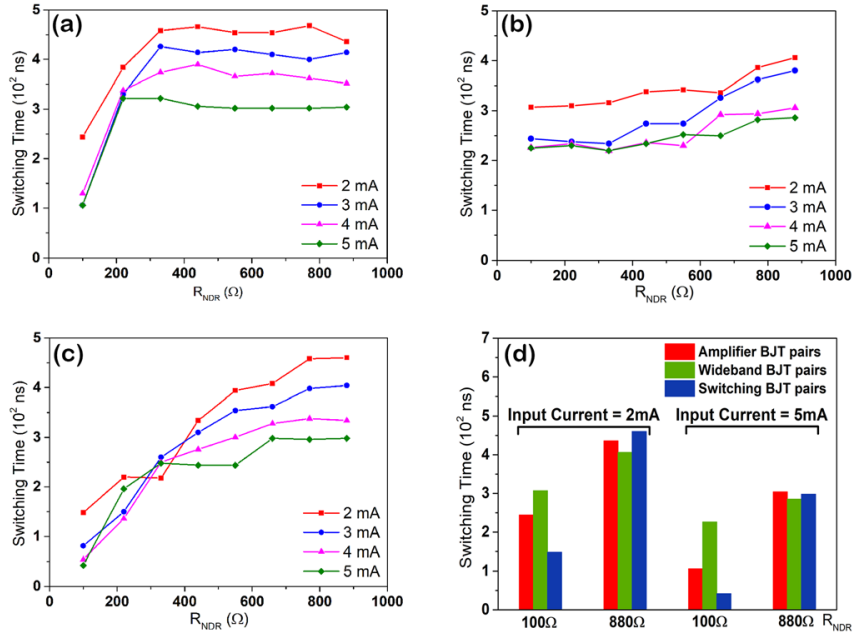

FIG. 2. Measured NDR switching time varies with $R_{N D R}$. In this measurement, different current pulses are applied, and a NDR with (a) amplifier BJT pairs, (b) wideband BJT pairs, and (c) switching BJT pairs is used. (d) The comparison of the NDR switching time with amplifier BJT pairs, wideband BJT pairs, and switching BJT pairs. The $R_{N D R}$ values are $100 \Omega$ and $880 \Omega$, and the applied current pulses are $2 \mathrm{~mA}$ and $5 \mathrm{~mA}$, respectively.

defined by contact lithography, followed by a $\mathrm{CF}_{4}$ plasma-based dry etch. The electrodes are patterned using contact lithography followed by magnetron sputtering of Ti/Au $(20 \mathrm{~nm} / 50 \mathrm{~nm})$. The magnetic component and $\mathrm{VO}_{2}$ device are then connected by ultrasonic wire bonding to form the logic-memory device.

Figure 3(a) illustrates the schematic of the two-terminal $\mathrm{VO}_{2}$ device, and an abrupt change in resistivity of the device is observed when the current reaches critical current $I_{c}$ of $0.21 \mathrm{~mA}$ [Fig. 3(b)]. In
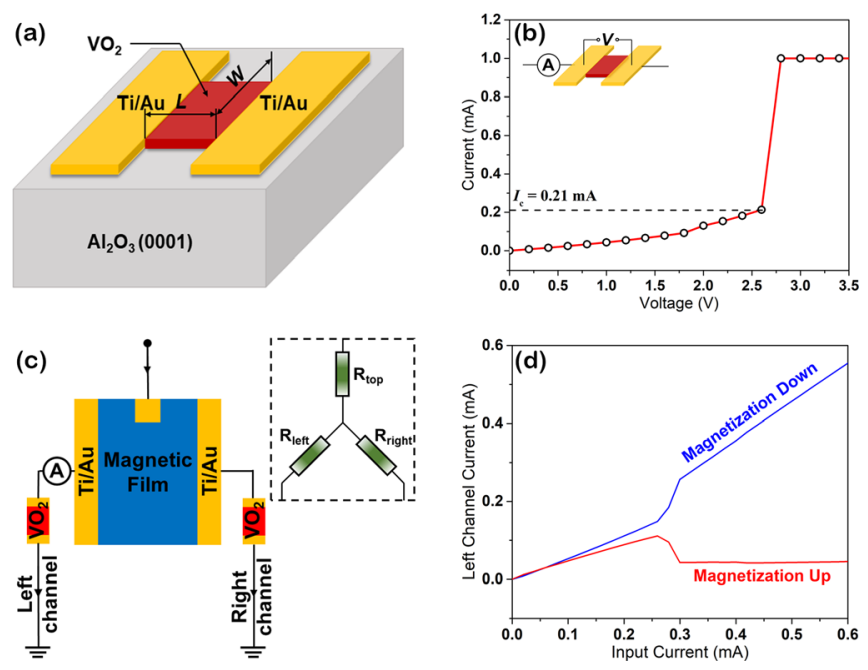

FIG. 3. (a) Schematic of a two-terminal $\mathrm{VO}_{2}$ device $(\mathrm{L}=3 \mu \mathrm{m} ; \mathrm{W}=5 \mu \mathrm{m})$. (b) $I-V$ curve of the two-terminal $\mathrm{VO}_{2}$ device. $\mathrm{A} 1 \mathrm{~mA}$-current compliance is used in the low-resistivity metallic state to limit the current and prevent thermal runaway in the $\mathrm{VO}_{2}$ device. (c) Schematics of the structure of our magnetic logic-memory device. (d) Left channel current of magnetization down and up. our magnetic device [Fig. 3(c)], the current imbalance caused by the AHE was dramatically enlarged by the IMT in the $\mathrm{VO}_{2}$ device [Fig. 3(d)]. According to Luo's analysis, ${ }^{2}$ the three-terminal magnetic component could be regarded as a Y-type resistor network, including $\mathrm{R}_{\text {Top }}$, $\mathrm{R}_{\text {Right }}$, and $\mathrm{R}_{\mathrm{Left}}$. If we consider the situation of magnetization up, electrons of spin up prefer to be deflected into the right side. $\mathrm{R}_{\text {Right }}$ is smaller than $R_{\text {Left, }}$ and so the current in the right channel $\left(I_{R}\right)$ is greater than the current in the left channel $\left(I_{L}\right)$. When the input current is small, both $I_{R}$ and $I_{L}$ are smaller than the critical current $I_{c}$ of the $\mathrm{VO}_{2}$ device $(0.21 \mathrm{~mA})$. With the input current increasing, $\mathrm{I}_{\mathrm{R}}$ first outweighs $\mathrm{I}_{\mathfrak{c}}$, and $\mathrm{VO}_{2}$ of the right channel enters the metallic state, leading to a dramatic drop of resistivity. Once the resistance of $\mathrm{VO}_{2}$ decreases, current bifurcation is amplified, leading to the dramatic increase in $I_{R}$ and decrease in $I_{L}$. The right and left channels are in the high current state (HCS) and the low current state (LCS), respectively. Moreover, the experimental results of a group of devices show that the differences in characteristics of our devices (e.g., critical current $I_{c}$ of $\mathrm{VO}_{2}$ devices, switching time, and output ratio of magnetic logic devices) are $<2 \%$, indicating that our device is stable.

Such a three-terminal magnetic device can be used for reconfigurable logic operations, ${ }^{2}$ with three magnetic components connecting in parallel as shown in Fig. 4(a). The magnetization direction down and up of the magnetic film (bit a, b) is defined as logic input " 1 " and " 0 "; meanwhile, the HCS and LCS are defined as logic output " 1 " and "0." Because of the AHE, the resistances of the left channel $\left(\mathrm{R}_{\mathrm{Left}}\right)$ and right channel $\left(\mathrm{R}_{\text {Right }}\right)$ are determined by the magnetization configuration of three magnetic bits. When bit $c$ is logic " 0 ," $\mathrm{R}_{\text {Left }}<\mathrm{R}_{\text {Right }}$ only for logic input ("1," "1") and $\mathrm{R}_{\text {Left }}>\mathrm{R}_{\text {Right }}$ for the other logic inputs. Therefore, the left channel was in the HCS (logic output " 1 ") only for logic input ("1," "1") and in the LCS (logic output " 0 ") in other logic inputs, which is the logic operation of AND. Meanwhile, current states of the right channel were the opposite to those of the left channel, which is the logic operation of NAND [Fig. 4(c)]. When bit $\mathrm{c}$ is logic " 1 ," $\mathrm{R}_{\text {Left }}>\mathrm{R}_{\text {Right }}$ only for logic input ("0," "0") and $\mathrm{R}_{\text {Left }}<\mathrm{R}_{\text {Right }}$ for the other logic inputs. The relationship between the current states of left and right channels and logic inputs is the logic relationship of OR and NOR, respectively [Fig. 4(c)].

For magnetic storage bits $\mathrm{d}$ and e, magnetization could be manipulated by current due to the built-in spin Hall effect offered by the Ta layer and SOT offered by the magnetic layer. ${ }^{12,13}$ The switching current of the magnetic component is approximately $0.5 \mathrm{~mA}$ with an axis-x magnetic field of $10 \mathrm{mT}$, which is smaller than the HCS and greater than the LHS when the applied current is $0.7 \mathrm{~mA}$. Hence, as shown in Fig. 4(a), when the applied current was $0.7 \mathrm{~mA}$, the channel current for logic " 1 " would switch the magnetization from up to down (write logic "1"), while the channel current for logic " 0 " would remain the magnetization up (write logic "0"). Logic operations and writing logic output into magnetic bits were finished at the same time, indicating that a logic device and nonvolatile memory were integrated. Moreover, the output ratio in our logic-memory device is greater than $1000 \%$ [Fig. 4(b)], making our device satisfy both reliable output and a low work magnetic field.

In addition, the switching time of the $\mathrm{VO}_{2}$ device in logic operations was measured to be only $\sim 7.5 \mathrm{~ns}$ [Fig. 4(d)], much shorter than that of the NDR. Moreover, unlike the dramatic variation of the NDR switching time with input current, the switching time of the $\mathrm{VO}_{2}$ device is insensitive to applied current. Therefore, our magnetic logic- 

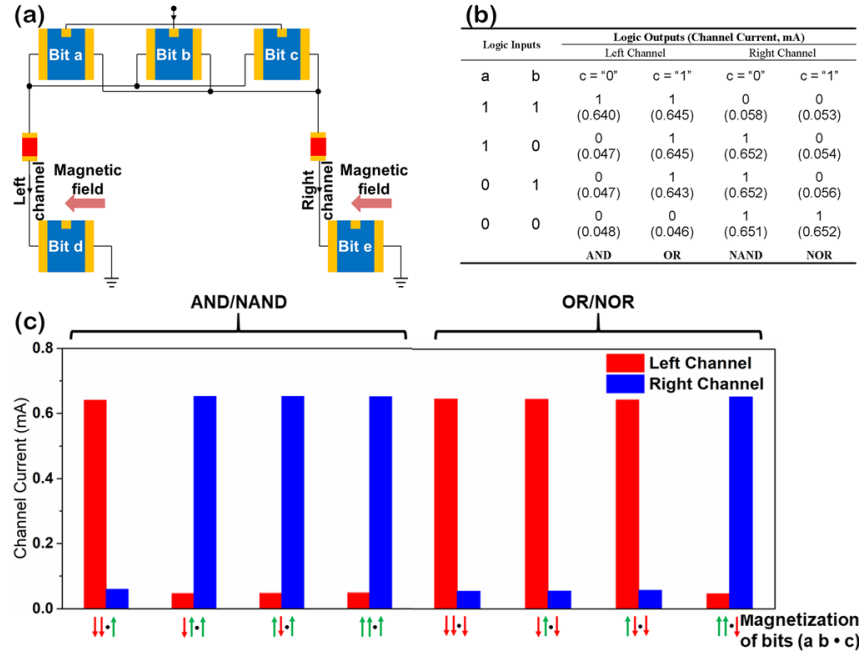

(d)

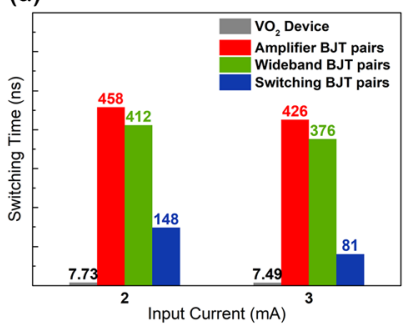

(e)

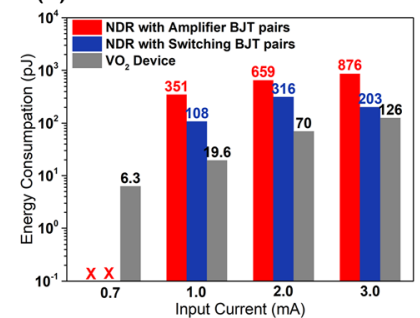

FIG. 4. (a) Schematics of the magnetic logic device with two magnetic output logic bits. (b) Truth table of magnetic logic. (c) Output channel currents of different magnetization configurations of three magnetic bits with an applied current of $0.7 \mathrm{~mA}$. (d) Comparison of the switching time for the $\mathrm{VO}_{2}$ device and NDR. (e) The calculated energy consumption of the NDR and $\mathrm{VO}_{2}$ device, and the red " $\times$ " represents that the NDR cannot work in this current.

memory simultaneously satisfies a reliable logic output ratio ( $>1000 \%)$, a low work magnetic field $(<10 \mathrm{mT})$, and excellent highfrequency performance (the switching time is $\sim 7.5 \mathrm{~ns}$ ). In addition, our measured switching time of $\mathrm{VO}_{2}$ includes delay caused by the connection with ultrasonic wire bonding and printed circuit board ( $\sim 6.32 \mathrm{~ns})$, and so the actual switching times of our logic-memory device are $1.27 \mathrm{~ns}, 1.5 \mathrm{~ns}$, and $1.26 \mathrm{~ns}$, corresponding to the applied current of $0.7 \mathrm{~mA}, 2 \mathrm{~mA}$, and $3 \mathrm{~mA}$, respectively. Hence, by improving the connection and using more suitable IMT materials, the highfrequency performance of our logic-memory device can be further improved (the details will be discussed later). The peak current is obtained under a voltage pulse whose width is switching time. The energy consumption is $I V t$, where $I$ is the current, $V$ is the voltage pulse amplitude, and $t$ is the pulse width. ${ }^{14}$ The energy consumption of our devices is shown in Fig. 4(e), and the minimum energy consumption of our device using a $\mathrm{VO}_{2}$ device (6.3pJ) is much smaller than that of a S-type NDR (351pJ).

In conventional computers, CMOS logic circuit and magnetic memory are separated, and so the processes of information transformation and transfer between these two units would consume a great amount of energy and time. Our logic-memory device could bridge the processor-memory gap in conventional computers, improving the performance of energy consumption and operation speed. Although the high-frequency performance of the semiconductor NDR in logic operations is poor, the switching time is dramatically reduced to $1 \mathrm{~ns}-10 \mathrm{~ns}$ by using IMT materials to replace the NDR. As the SOT induced magnetic switching in the $\mathrm{Ta} / \mathrm{CoFeB} / \mathrm{MgO}$ structure is $<180$ $\mathrm{ps},{ }^{12}$ the switching time of our device for logic operations and memory processes (IMT switching time + magnetic switching time) is $1 \mathrm{~ns}-10 \mathrm{~ns}$. The switching time might be further decreased to $\sim 10 \mathrm{ps}$ if we use $\mathrm{FeRh}^{15}$ or Co-doped $\mathrm{FeSi}^{16}$ to replace $\mathrm{VO}_{2}$ and use $\mathrm{Pt} / \mathrm{Ta} / \mathrm{Cu} /$ $\mathrm{Co} / \mathrm{Pt} / \mathrm{Ta}^{17}$ magnetic multilayers to replace $\mathrm{Ta} / \mathrm{CoFeB} / \mathrm{MgO}$ multilayers, which can outweigh that of CMOS circuits (100ps). ${ }^{14}$ In addition, our device needs less information transformation and transfer compared to CMOS circuits; consequently, our magnetic logicmemory device might dramatically improve the efficiency of computing with higher frequency, the feature of zero quiescent power, and "instant-on" performance.

In summary, in order to study the high-frequency performance of magnetic logic-memory devices consisting of $\mathrm{Ta} / \mathrm{CoFeB} / \mathrm{MgO}$ multilayers and S-type NDR components (complementary BJT circuits), we first investigated experimentally the switching time of NDR in logic operations, which was found to be hundreds of nanoseconds. Next, we developed a model to analyze the switching time theoretically and found that the switching time is limited by the internal turn-on property of BJT, and it is difficult to further reduce just the use of semiconductor NDR. Hence, we introduced IMT material $\mathrm{VO}_{2}$ into magnetic logic-memory devices and realized a magnetic logic-memory device with reliable output (output ratio $>1000 \%$ ), a low work magnetic field $(<20 \mathrm{mT})$, and excellent high-frequency performance (switching time$=1 \sim 10 \mathrm{~ns}$ ). Without information transformation between processors and memories, our device might dramatically improve the efficiency of computing.

See the supplementary material for (1) the details of the magnetic units and two-terminal $\mathrm{VO}_{2}$ device, (2) the NDR $I-V$ curve, and (3) the physical model of NDR.

This work was sponsored by the National Key R\&D Program of China (Grant No. 2017YFA0206202) and the National Science Foundation of China (Grant No. 11674190). The authors thank the Beijing National Center for Electron Microscopy for providing research facilities.

\section{DATA AVAILABILITY}

The data that support the findings of this study are available from the corresponding author upon reasonable request.

\section{REFERENCES}

${ }^{1}$ T. Ikenaga and T. Ogura, IEEE Trans. Comput. 47(7), 788 (1998).

${ }^{2}$ Z. Luo, Z. Lu, C. Xiong, T. Zhu, W. Wu, Q. Zhang, H. Wu, X. Zhang, and X. Zhang, Adv. Mater. 29(4), 1605027 (2017).

${ }^{3}$ D. Bhowmik, L. You, and S. Salahuddin, Nat. Nanotechnol. 9(1), 59 (2014); P. Chuang, S.-C. Ho, L. W. Smith, F. Sfigakis, M. Pepper, C.-H. Chen, J.-C. Fan, J. P. Griffiths, I. Farrer, H. E. Beere, G. A. C. Jones, D. A. Ritchie, and T.-M. Chen, ibid. 10(1), 35 (2015); J. A. Currivan-Incorvia, S. Siddiqui, S. Dutta, E. R. Evarts, J. Zhang, D. Bono, C. A. Ross, and M. A. Baldo, Nat. Commun. 7(1), 10275 (2016).

${ }^{4}$ S. Joo, T. Kim, S. H. Shin, J. Y. Lim, J. Hong, J. D. Song, J. Chang, H.-W. Lee, K. Rhie, S. H. Han, K.-H. Shin, and M. Johnson, Nature 494(7435), 72 (2013); 
Z. Luo, C. Xiong, X. Zhang, Z.-G. Guo, J. Cai, and X. Zhang, Adv. Mater. 28(14), 2760 (2016).

${ }^{5} \mathrm{~L}$. Chua, Y. Juebang, and Y. Youying, IEEE Trans. Circuits Syst. 32(1), 46 (1985).

${ }^{6}$ M. Imada, A. Fujimori, and Y. Tokura, Rev. Mod. Phys. 70(4), 1039 (1998).

7T. M. Rice, H. Launois, and J. P. Pouget, Phys. Rev. Lett. 73(22), 3042 (1994).

${ }^{8}$ W. A. Vitale, E. A. Casu, A. Biswas, T. Rosca, C. Alper, A. Krammer, G. V. Luong, Q. T. Zhao, S. Mantl, A. Schuler, and A. M. Ionescu, Sci. Rep. 7, 355 (2017).

${ }^{9}$ Y. Zhou, X. Chen, C. Ko, Z. Yang, C. Mouli, and S. Ramanathan, IEEE Electron Device Lett. 34(2), 220 (2013).

${ }^{10} \mathrm{~F}$. Shoucair and L. Trajkovic, "Analysis and simulation of simple transistor structures exhibiting negative differential resistance" (EECS Department, UC Berkeley, Berkeley CA, 1999).

${ }^{11}$ L. O. Chua and Y. W. Sing, IEE J. Electron. Circuits Syst. 3(1), 5 (1979).
${ }^{12}$ L. Liu, C.-F. Pai, Y. Li, H. W. Tseng, D. C. Ralph, and R. A. Buhrman, Science 336(6081), 555 (2012).

${ }^{13}$ I. M. Miron, K. Garello, G. Gaudin, P.-J. Zermatten, M. V. Costache, S. Auffret, S. Bandiera, B. Rodmacq, A. Schuhl, and P. Gambardella, Nature 476(7359), 189 (2011).

${ }^{14}$ Y. Mo, E. Skafidas, R. Evans, and I. Mareels, "A 40 GHz Power Efficient Static CML Frequency Divider in $0.13-\mu \mathrm{m}$ CMOS Technology for High Speed Millimeter-Wave Wireless Systems" in 4th IEEE International Conference on Circuits and Systems for Communications (IEEE, 2008).

${ }^{15}$ L. H. Lewis, C. H. Marrows, and S. Langridge, J. Phys. D: Appl. Phys. 49(32), 323002 (2016).

${ }^{16}$ N. Y. Xie, G. Z. Xie, X. Gao, L. J. Ye, L. Yang, C. Xiong, and J. Chen, J. Mater. Sci.-Mater. Electron. 30(19), 18065 (2019).

${ }^{17}$ K. Jhuria, J. Hohlfeld, A. Pattabi, E. Martin, A. Y. Arriola Cordova, X. Shi, R. Lo Conte, S. Petit-watelot, J. C. Rojas-Sánchez, G. Malinowski, S. Mangin, A. Lemaître, M. Hehn, J. Bokor, R. B. Wilson, and J. Gorchon, "Picosecond Spin Orbit Torque Switching," arXiv preprint arXiv:1912.01377 (2019). 